\title{
Air quality photochemical study over Amazonia Area, Brazil
}

\section{Lucio Silva de Souza* and Luiz Landau}

Department of Civil Engineering, Alberto Luiz Coimbra Institute Graduate School and Research in Engineering, Federal University of Rio de Janeiro - PEC-COPPE-UFRJ, Brazil, Centro de Tecnologia, Bloco I2000, Caixa Postal 68552, 21949-900, Rio de Janeiro, Brazil

and

Brazilian National Institute of Meteorology - INMET, 6th district of Meteorology,

Rio de Janeiro - Brazil

E-mail: luciodesouza@gmail.com

E-mail: landau@lamce.coppe.ufrj.br

${ }^{*}$ Corresponding author

\section{Nilton Oliveira Moraes}

Department of Mechanical Engineering,

Alberto Luiz Coimbra Institute Graduate School and Research in

Engineering,

Federal University of Rio de Janeiro - PEM-COPPE-UFRJ,

Centro de Tecnologia,

Bloco G, 21945-970, Brazil

E-mail: moraes.nilton@gmail.com

\section{Luiz Claudio Gomes Pimentel}

Department of Mechanical Engineering,

Alberto Luiz Coimbra Institute Graduate School and Research in Engineering,

Federal University of Rio de Janeiro - PEM-COPPE-UFRJ,

Centro de Tecnologia,

Bloco G, 21945-970, Brazil

and

Department of Meteorology,

IGEO, Federal University of Rio de Janeiro - UFRJ, CCMN, Brazil

E-mail: pimentel65@gmail.com 


\begin{abstract}
This work presents the results of a photochemical modelling system composed of MM5-SMOKE-CMAQ on Brazilian Amazonia area, been a pioneered implementation task. These results focus on the biogenic and biomass burning emissions and the impact of these emissions on regional air quality. Global and local anthropogenic emissions data, satellite-derived biomass burning inventories, and biogenic emissions calculated with MEGAN model were used to estimate emissions over the domain. The goal is the development of air quality model input data for CMAQ. Results are consistent with $\mathrm{O}_{3}$ formation theory and $\mathrm{CO}$ hotspot concentration matches with the geographic area of biomass burning.
\end{abstract}

Keywords: Amazonia photochemical air quality; SMOKE emissions processing; CMAQ forecast for Brazil; Amazonia area; photochemical air quality; SMOKE-CMAQ models.

Reference to this paper should be made as follows: Silva de Souza, L., Landau, L., Moraes, N.O. and Pimentel, L.C.G. (2012) 'Air quality photochemical study over Amazonia Area, Brazil', Int. J. Environment and Pollution, Vol. 48, Nos. 1/2/3/4, pp.194-202.

Biographical notes: Lucio Silva de Souza obtained his Bachelor in Meteorology Degree from the Federal University of Rio de Janeiro (UFRJ) in 2001, and his (MSc 2004) and PhD (2010) Degrees from the Alberto Luiz Coimbra Institute - Graduate School and Research in Engineering (COPPE/UFRJ) in Civil Engineering, with focus $\mathrm{n}$ Atmospheric Sciences. Nowadays de Souza works for the Brazilian National Institute of Meteorology (INMET) and is a substitute professor at the Rio de Janeiro State University, School of Geography. His research interests are air quality and atmospheric modelling, monitoring, forecasting and their impact on climate changes.

Luiz Landau graduated in Civil Engineering from the Pontificia Universidade Catolica do Rio de Janeiro-PUC/RJ in 1973, and obtained his MSc (1976) and $\mathrm{PhD}$ (1983) Degrees in Civil Engineering from the Federal University of Rio de Janeiro. He is currently a professor at the Alberto Luiz Coimbra Institute - Graduate School and Research in Engineering, Federal University of Rio de Janeiro. His research interests are in the areas of computational methods in engineering.

Nilton Oliveira Moraes has a Degree in Meteorology from the Federal University of Rio de Janeiro (2005) and an MSc Degree in the Mechanical Engineering programme PEM/COPPE (2008). He has experience in geosciences, with an emphasis on forecasting, synoptic meteorology, atmospheric modelling and air quality and micrometeorology (urban heat island).

Luiz Claudio Gomes Pimentel received his Bachelor's Degree in Chemical Engineering from the Federal University of Rio de Janeiro (UFRJ) in 1987. $\mathrm{He}$ received his MSc and PhD Degrees from the Federal University of Rio de Janeiro in mechanical engineering in the field of turbulent fluid mechanics and thermal engineering. His research interests are in the areas of air quality modelling, atmospheric boundary layers and atmospheric modelling. 


\section{Introduction}

Air quality forecasting over the Brazilian Amazonia Area is still a theme of uncertainties. The lack of emission inventories and air quality monitoring networks for that area still remains a problem for those interested in studying this theme. On the other hand, the Amazonia has been experiencing a stronger change process due to economic growth. The main metropolitan areas are Manaus and Belem, and only these two urban complexes have a total population of 4,152,000 inhabitants, while in the total legal Brazilian Amazonia area (composed of 9 states), the population comprises around 20 million inhabitants (IBGE, 2000).

Changes in land use patterns because of deforestation (controlled or not) compose one of the main reasons for that scenario changing. The so called 'arc of deforesting' is a proof of those changes and has a stronger correlation with the agriculture and livestock expansion for that region. Figure 1 shows the 'arch' that has been under control by the authorities; it is nowadays called the "arch of sustainable development".

In Amazonia, there's a petroleum and natural gas plant in the southwest of Amazonas State and a refinery in the Manaus metropolitan area. These facilities are responsible for feeding that area with fossil fuels, and altogether with land use changes due to biomass burning and economic growth, confirm the need for air quality studies covering Brazil's North Region.

The MM5-SMOKE-CMAQ system is implemented for Brazil with a coarse horizontal resolution of $37 \mathrm{~km}(165 \times 165$ points $)$, and 23 vertical sigma levels. Details of SMOKE and CMAQ components can be found at the Community Modelling and Analysis System website (cmascenter, 2010). The SMOKE model (smoke-model, 2010) was filled with biomass burning data from the GFED inventory, part of GEIA (www.geiacenter.org). Biogenic emissions were obtained from the MEGAN model (Guenther et al., 2006), with a resolution of $1 \mathrm{~km}$. These two emission components might be governing the in charge for photochemistry in Amazonia, particularly if the simulations are focused on a regional scale. Shipping emissions, main point sources and national roads were inserted from the GIS global database as well, and were treated as area sources through SMOKE.

Figure 1 Arch of Deforestation (2006) (see online version for colours)

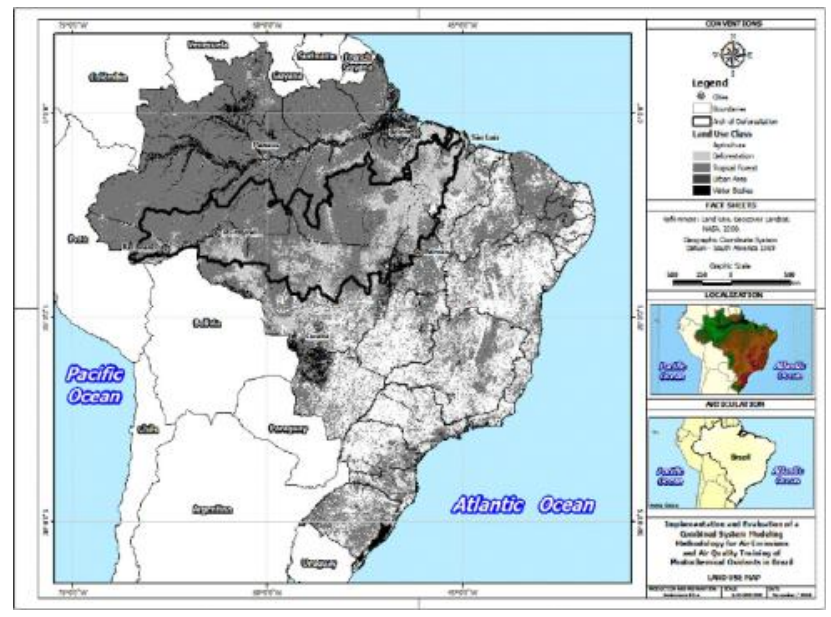




\section{Objectives}

The main objective of this work is focused on the implementation of the system composed of MM5-SMOKE-CMAQ models to Brazil and its surroundings. Additional objectives are discussion of the initial results for air quality photochemical forecast over the Brazil Amazonian area, as well as illustration of the emission inventories framework to perform that modelling for South America. The lack of a public national emission inventory and air quality-based ground monitoring network makes this work a challenge by adapting global to regional inventories and their assimilation to the SMOKE model. The problem identification of this task as a whole might also be considered as an objective.

\section{Methodology}

The 2005 dry season was the most intense of the last 50 years over the Amazonia area. This season has been linked to the anomalies in Sea Surface Temperature (SST) that were some of the main causes of the intense hurricane season over the North Atlantic and Caribbean area that year (CLIMANALISE, 2005). This SST anomaly implied a very unstable atmosphere, driven by upward vertical motion in the Northern Atlantic and, as a response to that upward motion, the downward motion was directed at Northern Brazil. This motion blocked the convection mechanism and the dominant rainfall regimen over that area, causing significant problems to the people and environment of that area.

The PSU/NCAR mesoscale meteorological 5th generation model (MM5) is used to feed the SMOKE-CMAQ system with hourly meteorological data. The MM5 outputs are processed throughout the Meteorology Chemistry Interface Processor (MCIP) that performs the changes needed to the original MM5 outputs for use in SMOKE-CMAQ. This tool is available together with SMOKE and CMAQ (www.cmascenter.org). The MM5 is run with GFS-NCEP global data $(1 \times 1)$, with a mother domain horizontal resolution of $37 \mathrm{~km}$ and two nested grids (12,3 and 4,1 km); the outer grid is centred at the geographic South American centre, while the two other domains are at the Brazilian Amazonian Area. The smaller is centred in the petroleum and natural gas facility in the southwest of the Amazonian state (BOGPM), the biggest petroleum and natural gas facility in the Brazilian Northern Region. This MM5 run is composed of 24 vertical layers, of which 8 are at the boundary layer. The MM5 parameterisations schemes are displayed in Table 1.

Table 1 MM5 Parameterisations

\begin{tabular}{ll}
\hline Cumulus & Grell (1993) domain 1 and $2^{1}$ \\
Clouds Microphysics & Dudhia (1989) \\
Radiation & Dudhia (1989) \\
Landuse & Landuse model NOAH LSM (Chen e Dudhia, 2001) \\
PBL & Mellor e Yamada (1974, 1982) \\
\hline
\end{tabular}

Usually the best map projection for the South American tropical area is Mercator. The models SMOKE and CMAQ didn't run well with Mercator, presenting numerical 
errors and inconsistencies. The solution to obtain initial SMOKE-CMAQ results was to run the system with Lambert Conformal projections. Further, the MM5 nested grids were not applied for SMOKE and CMAQ due to the same numerical errors linked to the projection, leaving only the $37 \mathrm{~km}$ grid as the domain able to show results.

The SMOKE model (www.smoke-model.org) is used to provide emission inventory data as input to the CMAQ model. Generally, SMOKE processes emissions from typical emission inventories formats into the format required by the CMAQ model (www.smoke-model.org). For this work, SMOKE was used to process emissions from the GFED biomass burning global emission inventory (www.geiacenter.org), with $1 \times 1$ horizontal resolution, year base 2005 and a total monthly mean extracted and processed to daily basis. This data processing is done with support programs available altogether with SMOKE and CMAQ at the web portal (www.cmascenter.org). In the case of national mobile road emissions, these data were extracted from the GIS Brazil National Geographic Institute (IBGE, 2000) and the emission factors are informed according to the available GIS global database. The mobile sources are processed into a weekly basis, and the SMOKE holiday tool isn't set for this run. Shipping traffic and emissions are extracted from the GIS information base, and are also processed on a weekly basis. In all SMOKE steps, meteorological information processed through MCIP must be provided before running the merge script, the one that makes the processing for all types and creates gridded emissions in moles/s as required by the CMAQ model. The chosen chemical mechanism is CB05 and at this moment, the chemical mechanism is not the focus of this work. Shipping emissions and the main national Brazilian roads (only federal roads) were also provided as area sources for the SMOKE model.

The biogenic emissions were extracted from the MEGAN model (Guenther et al., 2006) and the outputs were inserted into SMOKE with a horizontal resolution of $1 \mathrm{~km}$. When biogenic emissions come from the MEGAN model, the SMOKE model doesn't process this category of emissions because the outputs are ready for CMAQ input and SMOKE works on the adjustments of the spatial resolution from MEGAN to the one set at the SMOKE-CMAQ grid, keeping the constancy and meteorological aspects to the new resolution. Meteorological information for MEGAN is also required, and is processed at the same manner as for the SMOKE model with MCIP converser.

The CMAQ model (Byung et al., 1998) is run with a $37 \mathrm{~km}$ horizontal resolution centred at South America's geophysical centre, exactly as for MM5 and SMOKE. The task for running is not as complex as it is for SMOKE and its support programs, because once the emissions files from SMOKE are available, CMAQ trends to run properly with correct shell scripts manipulation and chemical mechanism choice through the modeller. In our study, the focus on CMAQ is to stress that this model might be used for South America, once the numerical structural problems (map projections, compilers, etc.) in the code are solved. The CMAQ initial conditions were run to use the default profile Initial Conditions (IC's), the plume in grid option was not set, the meteorology was set with $3 \mathrm{D}$ met fields as requested to nested grids and the mechanism conversion was set according to CB05. Boundary Conditions (BC's) were also run with the default profile input, including plume in grid profiles from IC's dynamics and concentration generation, with otherwise the same settings as described for IC's. The photolysis rates are run on the JPROC program and set as the meteorological information has been disposed from MM5-MCIP. The JPROC performs the photolysis rates, which are highly influenced by meteorological conditions. No global chemical model is used to feed the 
37 domain borders at this work because the grid limits are at the ocean, but it is important to remark that this task is still under development.

The main problems linked to this system became apparent when converting the meteorological data in the MCIP converser to feed SMOKE with meteorological data. There was a structural problem with the MCIP (version 3.5, beta) code that didn't support the Mercator recommended projections for tropical areas, raising the need to configure the model with Lambert Conformal projections, because it would not proper for our area of interest. It should be noticed that the same problem was found when assimilating the GIS information into SMOKE for the surf zone coastal area. Nowadays, after a joint effort with the MCIP staff, the new MCIP version (3.6) supports Mercator projections, (http://www.cmascenter.org/help/model_docs $/ \mathrm{mcip} / 3.6 /$ ReleaseNotes) but the problem persists in SMOKE and CMAQ.

\section{Results}

The discussion starts with the emission results for the day August 5th, 2005. The maps in Figure 2 show the highest emissions obtained for this day in moles/second ( $\mathrm{mol} \mathrm{s}^{-1}$ ), the required unit for CMAQ input, and are based on the biogenic emissions extracted from the MEGAN model.

Figure 2 Megan - Biogenic highest Emissions $\left(\mathrm{mol} \mathrm{s}^{-1}\right.$ ) for Isoprene: (a) 1600 GMT; (b) 1700 GMT; (c) CO-1600 GMT and (d) CH4 Greenwich Medium Time - GMT

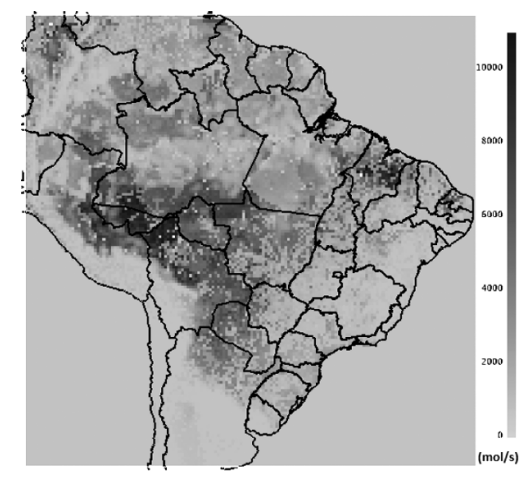

(a)

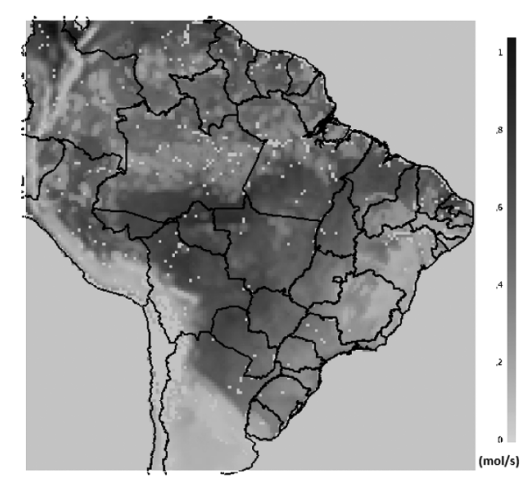

(c)

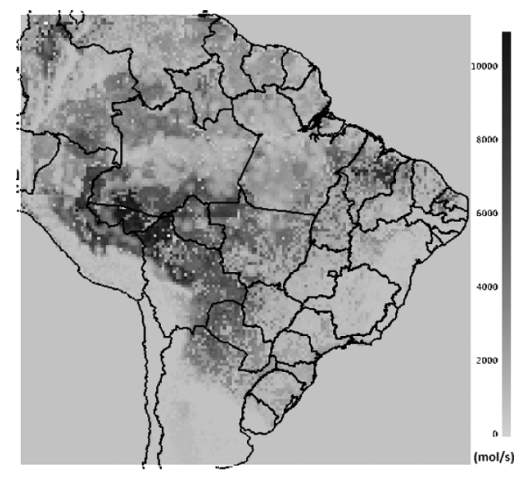

(b)

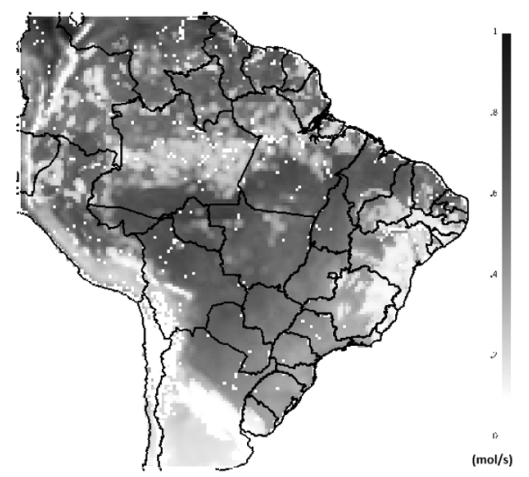

(d) 
The emissions at Figure 2 show that biogenic emissions in South America have a stronger dependency on sunlight, and the highest concentrations are observed in the early afternoon, local time. For $\mathrm{CH}_{4}$ and $\mathrm{CO}$, it is possible to observe emission profiles in the east and northeast Brazilian areas that are not observed when looking at isoprene emissions. In both cases, it is possible to identify some peaks in emissions in the south Amazonia area. Another important characteristic of this type of emission is the gap in the total estimate of emitted isoprene (thousands of $\mathrm{mol} \mathrm{s}^{-1}$ ) and $\mathrm{CO}$ and $\mathrm{CH}_{4}$ (units of $\mathrm{mol} \mathrm{s}^{-1}$ ).

Considering the anthropogenic emissions, it is necessary to remark the lack of a national emission inventory, reinforcing that the results come from processing global data into a regional scale. Figure 3 shows the highest emission extracted from the SMOKE model in $\left(\mathrm{mol} \mathrm{s}^{-1}\right)$, as required in CMAQ. CO is shown due to its importance when considering emission from biomass burning, especially in the south and southwest Amazonian areas.

Figure 3 SMOKE highest Emissions $\left(\mathrm{mol} \mathrm{s}^{-1}\right)$ for CO Greenwich Medium Time - GMT

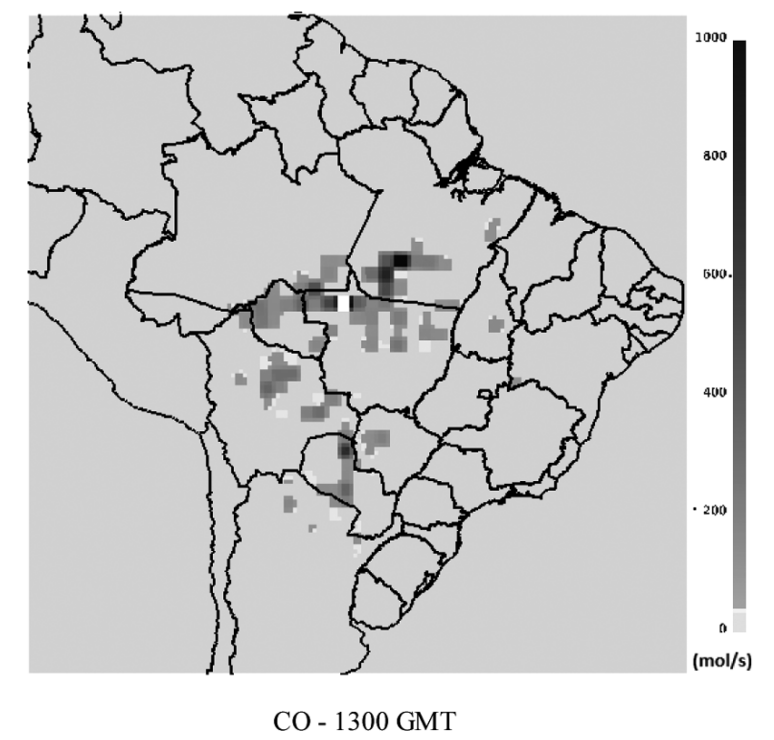

The results shown in Figure 4 correspond to CMAQ $\mathrm{O}_{3}$ output for the same day (August 5th 2005) at highest concentration forecast hours (1400 and 1600 GMT), and it is possible to identify peaks in the southern Amazonia, area during the afternoon, as expected, both from the theory and from the emission dataset used to perform the run.

The possibilities for comparison of $\mathrm{O}_{3}$ outputs are linked to the remote sensing data, the only available tool for this task in Amazonia. As the results from remote sensing for ozone were unreliable, this activity is performed by comparing the peak concentrations forecasted through the system for $\mathrm{CO}$ with MOPITT data (Figure 5).

An evaluation of Figure 5 shows that the highest forecasted $\mathrm{CO}$ concentrations are at the same geographic area as the satellite image shows. The gap between the values forecasted and registered are due to the output representation for only one hour, while the satellite represents the total daily amount surface registered. By extrapolating the hourly concentrations, it is easy to identify that the CMAQ results are underestimating the 
concentrations according to the satellite estimates, but in a similar order of magnitude. This difference makes sense once we consider that only global biomass burning and global GIS data are used as input for the runs.

Figure 4 CMAQ outputs (ppmV) for Ozone - Greenwich Medium Time - GMT cmaq $\mathrm{O}_{3}$ output (ppmV): (a) 1400 and (b) 1600

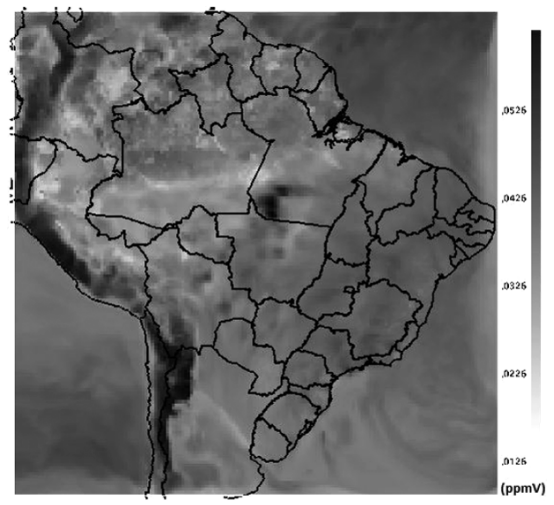

(a)

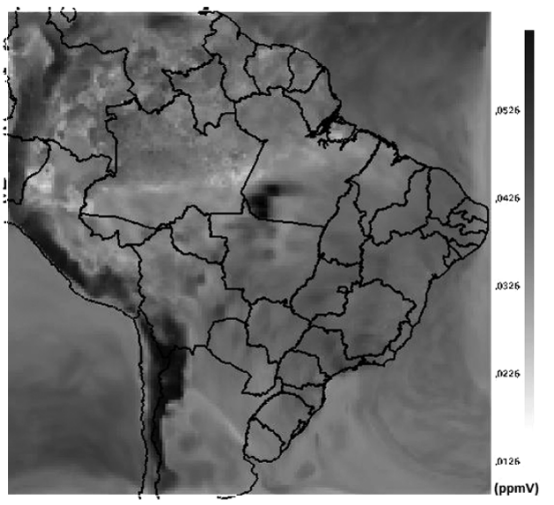

(b)

Figure 5 CMAQ outputs (ppmV) for CO: (a) - Greenwich Medium Time (GMT) Satellite images from MOPITT and (b) August 5th 2005 (see online version for colours)

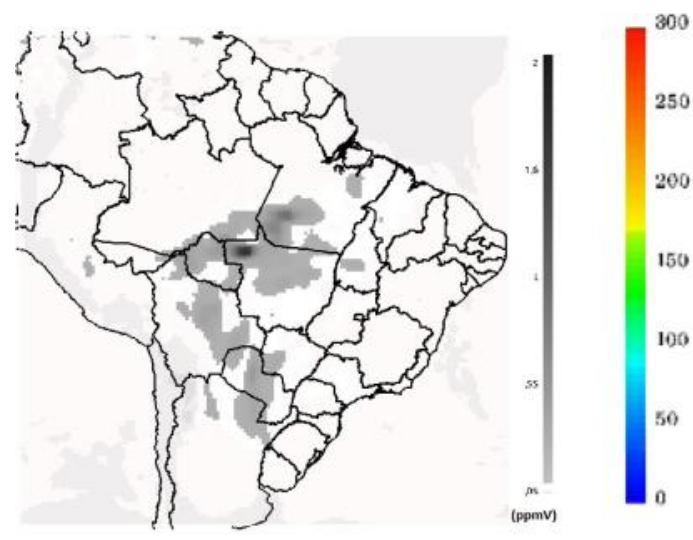

(a)

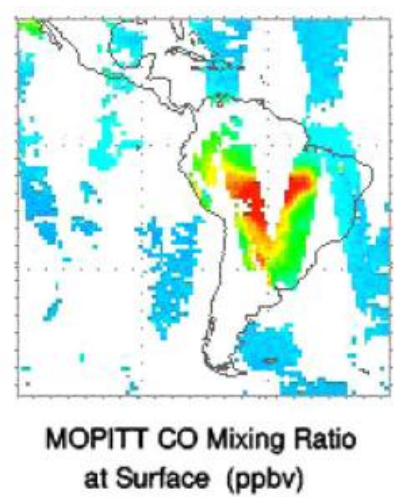

(b)

\section{Conclusion}

The lack of air quality-based ground monitoring stations and national/local inventories imposes a limitation on the evaluation of the results presented in this paper.

The system MM5/SMOKE/CMAQ has been implemented in Brazil with grids that make it possible to apply this computing tool as it is applied in North America, with a large domain covering almost all of the continent. The emission data were extracted from global bases and GIS datasets and biogenic data were extracted from the MEGAN model. Apart from structural errors with projections, the system is able to represent what is expected from the theory, and the results are in agreement with the location of 
some hotspots satellite data, especially for $\mathrm{CO}$. When trying to compare the $\mathrm{O}_{3}$ results, the available remote sensing data weren't proper to perform that type of comparison, as they were far from ideal. This system needs improvements in the emissions to better generate results, as more remote sensing data analysis and processing is necessary to better compare the numerical results for the Amazonian area. The system might, however, be used for other Brazilian areas to perform photochemical air quality modelling studies.

\section{References}

Byun, D.W. and Ching, J.K.S. (1998) Science Algorithms of the Epa Models-3 Community Multiscale Air Quality (Cmaq) Modelling System, U.S. EPA/600/R-99/030.

CLIMANALISE (2005) Boletim de Monitoramento e Análise Climática, (http://www6.cptec.inpe.br/revclima/boletim/) Vol. 20, Agosto 2005. Accessed between January 2005 and March 2010.

CMASCENTER (2010) Community Modeling and Analysis System, (http://www.cmascenter.org/). Accessed between 2006 and April 2010.

Guenther, A., Karl, T., Harley, P., Wiedinmyer, C., Palmer, P. and Geron, C. (2006) 'Estimates of global terrestrial isoprene emissions using MEGAN (Model of Emissions of Gases and Aerosols from Nature)', Atmospheric Chemistry and Physics, Vol. 6, pp.3181-3210.

IBGE (2000) Brazilian Institute of Geography and Statistics (http://www.ibge.gov.br/home/) Accessed between January 2005 and March 2010.

SMOKE-MODEL (2010) Sparse Matrix Operator Kernel Emissions, (http://www.smokemodel.org/index.cfm). Accessed between 2006 and April 2010.

\section{Note}

${ }^{1}$ Not applied for horizontal resolutions under $10 \mathrm{~km}$. 